\title{
Image Set Classification Based on Cooperative Sparse Representation
}

\author{
Peng Zheng ${ }^{a}$, Zhong-Qiu Zhao ${ }^{a *}, \mathrm{Jun} \mathrm{Gao}^{a}$, Xindong $\mathrm{Wu}^{a, b}$ \\ ${ }^{a}$ College of Computer Science and Information Engineering, Hefei University \\ of Technology, Hefei 230009, China \\ ${ }^{b}$ Department of Computer Science, University of Vermont, Burlington, USA
}

\begin{abstract}
Image set classification has been widely applied to many real-life scenarios including surveillance videos, multi-view camera networks and personal albums. Compared with single image based classification, it is more promising and therefore has attracted significant research attention in recent years. Traditional (forward) sparse representation (fSR) just makes use of training images to represent query ones. If we can find complementary information from backward sparse representation (bSR) which represents query images with training ones, the performance will be likely to be improved. However, for image set classification, the way to produce additional bases for bSR is a problem concerned as there is no other bases than the query set itself. In this paper, we extend cooperative sparse representation (CoSR) method, which integrates fSR and bSR together, to image set classification. In this process, we propose two schemes, namely 'Learning Bases' and 'Training Sets Division', to produce the additional dictionary for bSR. And different from previous work, our work considers scene classification as a problem of image set classification, which will provide a new insight for scene classification. Experimental results show that the proposed model can obtain competitive recognition rates for image set classification. By combining information from
\end{abstract}

*Corresponding author. Email: z.zhao@hfut.edu.cn

Preprint submitted to Journal of LATEX Templates

May 13, 2016

(C) 2016. This manuscript version is made available under the Elsevier user license http://www.elsevier.com/open-access/userlicense/1.0/ 
these two opposite SRs, better results can be achieved. Also the feasibility for the formulation of image set classification on scene classification is validated.

Keywords: sparse representation, image set classification, dictionary learning, scene classification

\section{Introduction}

Traditionally, we solve recognition problems in computer vision mainly based on single images, where our task is to identify query image one by one [1, 2]. With the increment of available video cameras and large capacity storage media,

5 in a number of real-life applications such as security and surveillance systems, personal albums and multi-view camera networks, we can collect multiple images of a person or an object. To recognize these multiple images, we usually formulate it as an image set classification problem where a set of images of the same unknown object are supplied. And there has been growing interest in this field in recent years 3 [10.

Usually appearance variations in images are caused by background switches, viewpoint variations, illumination condition changes, non-rigid deformations, occlusions and disguise. Compared with single image based classification, methods based on image sets are expected to give better performance. The reason is that they can effectively handle a wide range of these appearance variations and allow the decision process to be based on comparisons between the most similar pairs of query and gallery images. However, in spite of the providing of numerous images about the same object under different appearance variations, it is confronted with many challenges to make effective use of this data and conduct a reliable decision by integrating the information from all the images of the sets. Therefore, many researchers proposed different techniques and tried to find suitable representations which can efficiently and accurately model the intra similarities within images of an image set and the inter differences between different sets. For example, the methods in [3 [5, 11] use subspaces to model 
image sets, and set representative exemplars (generated from affine hull/convex hull) are used in [6, 8] for image set representations. The mean of the set images is used for set representation in [8, 12, 13] and image sets are represented as a point on a manifold geometry in [7, 14].

Inspired by the findings that natural images can be generally coded by structural primitives (e.g., edges and line segments) similar to the phenomenon in simple cell receptive fields [15], sparse representation chooses a small number of atoms from an over-complete dictionary to represent natural images. As it has low reconstruction errors and is robust to noise, occlusion and corruption, it is successfully applied to face recognition [16], image classification [17] and video processing [18, 19]. And authors in [8, 20] adopted this theory to solve problems in image set classification. They achieved the goal by representing different sets with image samples of the same set as well as their affine hull model and calculating set-to-set distance with most relevant samples or subspaces obtained through iterative optimization. Meanwhile, G. Ortiz et al. proposed Mean Se-

40 quence Sparse Representation Classifier (MSSRC) by adding a strict temporal constraint to the $\ell_{1}$-minimization. It takes advantages of individual frames in a face track to reconstruct an identity together and reduces the optimization to a single minimization over the mean of the face track [13. On one hand, the methods in [8, 20] limit the representative samples to their respective sets, which

45 abandons the discriminant ability of sparse representation, and the brute-force way to combine different models results in a complex optimization function. On the other hand, [13] just uses the samples in training sets to represent the ones in query set, which does not make full use of the correlations between the training sets and query set, and the mean image usually can not represent the inter differences of each set well.

Recently, Zhao et al. proposed a cooperative sparse representation (CoSR) model, which integrates the information from both forward sparse representation (fSR) and backward sparse representation (bSR), for semi-supervised image annotation [21. Considering the situations that there are not enough samples ${ }_{55}$ provided in each set - namely relatively small set size, it is more difficult to 
recognize different objects based on limited information especially when the images are in low resolution. However, for the CoSR model, we can find out the most relevant concept for query set with fSR representing samples in query set by training sets at first. And then from an opposite direction, we can bridge the correlations between training sets and query set with bSR representing samples in training sets by query set. With the cooperation and complementarity between these two SRs, more valuable discriminant information can be explored even with fewer samples and made full use of to accomplish image set classification task better. This makes the CoSR model quite suitable for image set classification especially when the set size is small. Meanwhile, there are advantages such as relatively simple optimization procedure, more discriminant structures and fewer parameters for this model over the ones above.

To apply the CoSR model to image set classification, the main problems fall in the procedure of bSR. This is due to the fact that the images in the query set are all from the same concept, which are not discriminant enough as a dictionary to separate samples of different concepts well. Here 'discriminant' means that the sets with different class labels should be well separated and the samples within each set are compacted if the dictionary is well constructed. Also the relatively small set size is likely to 75 make the over-complete assumption of sparse representation invalid. Inspired by the works mentioned above, in this paper, we solve these problems by constructing additional bases for bSR via two schemes, namely 'Learning Bases' and 'Training Sets Division', and extend the CoSR model to the field of image set classification. Although these two schemes are different from each other, they can be considered as two strategies to stimulate sample distribution in real applications from different viewpoints. One makes use of existed samples in training sets while the other produces artificial discriminant atoms. Experimental results on several benchmark datasets exhibit the effectiveness of the proposed method.

At the same time, different from previous work limited to face identification and object categorization, we formulate scene classification, which usually owns 
relatively larger inter differences as well as more complex formation and is a rather difficult problem, as an image set classification problem. This will give a new alternative solution for scene classification task from a different viewpoint.

90 Also experimental results demonstrate that the formulation is reasonable and a better performance could be obtained.

The main contributions of this paper are as follows:

1) We extend the CoSR model to image set classification, which provides ways to make use of complementary information from both fSR and bSR to improve 95 identification performance.

2) We take two methods, namely 'Learning Bases' and 'Training Sets Division', to construct additional bases for bSR, which makes the model suitable for different situations.

3) We regard scene classification task as a member of image set classification and validate its reasonability through experiments.

The rest of this paper is organized as follows. Some related works are reviewed in Section 2 at first. In Section 3, the details of the original CoSR model are provided. Then two techniques to construct additional bases for bSR are introduced in Section 4. Experimental results and corresponding analyses on several benchmark datasets are provided in Section 5. At last, concluding remarks are presented in Section 6.

\section{Related Work}

To solve the problem of image set classification, many researchers have proposed a great deal of methods. According to the type of representations, existing methods can mainly be categorized into two classes, namely parametric-models and non-parametric-models. The parametric-models 22] use a certain statistical distribution model to formulate each image set and then adopt measurements such as KL-divergence to measure the similarity between two image sets. Their performance is greatly influenced by the statistical relationship between the query and training sets and a bad result will be produced if there does not exist 
a strong one.

The non-parametric models do not rely on the statistical relationship and represent an image set either by its representative exemplars or on a geometric surface. For methods which model a complete image set as a point on a geometric surface [4, 5, 7, 14, either of the following forms, namely a subspace, mixture of subspaces or a complex non-linear manifold, can be adopted to represent different sets. And accordingly different distance metrics are provided to cooperate with them. For those represented by a linear subspace, researchers determine the between-set distance by principal angles and measure the similarity between subspaces by the sum of the cosines of these principal angles. For image set representations on manifolds, many methods adopt different manifolds accompanied by suitable distance metrics such as geodesic distance [23], the Grassmann manifold with the projection kernel metric [24, and the Lie group of Riemannian manifold with the log-map distance metric [25]. To discriminate image sets on the manifold surface, different discriminant analysis methods were proposed for different set representations, including Discriminative Canonical Correlations (DCC) 3], Manifold Discriminant Analysis (MDA) [5], Graph Embedding Discriminant Analysis (GEDA) 7] and Covariance Discriminative Learning (CDL) 14]. These methods are computational efficient and compact. However, the representations do not necessarily encode all the information contained in each image set and can be sub-optimal with a loss of some information. For example, for image sets represented by a subspace, the amount of the retained information depends on the selected dimensions of the subspace.

In the meantime, among the methods which represent an image set by its representative exemplars, the simplest one should be the set mean [4] or adaptively learnt set samples [6, 8]. And accordingly the set-to-set distance is usually defined as the Euclidean distance between the set representatives. Cevikalp et al. modeled the set images with the affine hull or convex hull models and learned set samples from them. Then Affine Hull Image Set Distance (AHISD) or Convex Hull Image Set Distance (CHISD) was adopted to measure the similarities 
[6. However, the learned set samples could be visually very different from the rest of the images in the set. And the one proposed by $\mathrm{Hu}$ et al. 8 is quite relevant as well. The authors defined the Sparse Approximated Nearest Points (SANPs) through the regularization from the mean image and affine hull model of the corresponding sets and determined the set-to-set distance by searching for the closest points between sets via iterative optimization. As it adaptively chooses optimal samples to obtain the distance between sets, it is capable of handling intra set variations effectively. However, measuring the distances with certain samples will ignore structural information, which makes it highly prone to outliers. And the brute-force way to combine the affine hull representation and sparse representation results in a rather complex model (three representation terms, four parameters and four unknown variables). G. Ortiz et al. proposed a method named MSSRC in [13, which reduced the problem of solving sparse representation coefficients for samples in query set to a single minimization over the mean of the face track. It can be treated as the optimization version over forward sparse representation and categorized as a part of the CoSR model below. To solve these problems and explore more valuable information from the correlations between query set and training sets, we take the CoSR model proposed in 21] into consideration.

The CoSR model, which combines fSR representing testing images with training ones and the opposite bSR representing training samples with testing ones, is initially designed for image annotation. Whereas there is only the query set provided for image set classification, the semi-supervised assumption of the original model will be invalid, where all the samples of the testing data can be used as unlabeled samples to assistant the training of classifiers. So in this paper, we propose several schemes to construct discriminant additional bases to accomplish bSR and extend this model to image set classification. It should be noticed that the original CoSR model is a time-consuming iterative procedure via co-training, which tries to solve a two-class problem annotating each concept independently. However, image set classification is quite a different task from image annotation, which 
conducts reliable decisions based on image sets with multiple classes and has a relatively high requirement for efficiency. So the proposed schemes are of importance for the extension from image annotation to image set classification. Meanwhile, the advantages of the modified CoSR model for image set classification come in four folds:

1) the adoption of sparse coefficients as ownership probabilities avoids the calculation of one-to-one distances;

1852 ) the combination of these opposite SRs as the bridge for the query set and training sets will fuse the information of both to make better decisions, which is capable of exploring more valuable information and makes the model robust to the reduction on the set size;

3) different proposed schemes will make the model more stable and suitable for 190 different situations;

4) the optimization procedure is relatively simple and only a few parameters need to be considered.

Besides that, although there have already been many methods proposed to classify different scenes, such as the ones in [26-29], they just used the background and context information contained in a single testing image. As the information provided by only one testing sample is quite limited, it is hard for us to get a promising result. If we can explore more valuable information and abandon useless noise from more provided testing samples of the same concept, namely a set, we may obtain a better result. In fact, we usually face this kind of situation that many photographs are taken from the same scene by the tourists definitely. So in this paper, we accomplish scene classification task from a different viewpoint and try to model it as an image set classification problem. And the results on Scenes 15 dataset support the feasibility of our assumption. 


\section{Cooperative Sparse Representation}

In this section, we will give a detailed introduction for the original CoS$\mathrm{R}$ model for image annotation (here some minor changes have been added to change it from a two-class problem to multi-class problem). And it will be introduced with three parts, forward sparse representation (fSR), backward sparse representation (bSR) and the fusion of these two SRs.

\subsection{Forward Sparse Representation}

Given the training data $X=\left[X_{1}, X_{2}, \cdots, X_{c}\right] \in R^{d \times m}$ consisting of $c$ different classes and a testing image $y$, we can treat training data $X$ as a dictionary to linearly represent $y$ as follows:

$$
y=X \alpha+e
$$

where $e \in R^{d}$ is the signal noise in the dataset and $\alpha \in R^{m}$ is the reconstruction coefficients. According to the theory of sparse representation, minimizing the following objective function can solve the approximation problem in Eqn.(1):

$$
\min _{\alpha}\|y-X \alpha\|^{2}+\lambda\|\alpha\|_{1}
$$

where $\|*\|$ and $\|*\|_{1}$ represent $\ell_{2}$-norm and $\ell_{1}$-norm respectively. $\lambda$ is a parameter controlling the tradeoff between reconstruction residuals and the sparsity of the vector $\alpha$.

As each $X_{i}=\left[x_{i, 1}, x_{i, 2}, \ldots, x_{i, m_{i}}\right]$ consists of $m_{i}$ column-wise feature vectors, where $i=1,2, \ldots, c$ and $\sum_{i} m_{i}=m$, we can rewrite Eqn.(1) as follows

$$
\begin{aligned}
y & =\sum_{i=1}^{c} \sum_{j=1}^{m_{i}} \alpha_{i, j} x_{i, j}+e \\
& =\alpha_{1,1} x_{1,1}+\alpha_{1,2} x_{1,2}+\cdots+\alpha_{1, m_{1}} x_{1, m_{1}} \\
& +\cdots \cdots \\
& +\alpha_{c, 1} x_{c, 1}+\alpha_{c, 2} x_{c, 2}+\cdots+\alpha_{c, m_{c}} x_{c, m_{c}}
\end{aligned}
$$

Here $\alpha_{i, j}$ is the element in $\alpha$ associated with $x_{i, j}$ - the $j$-th sample in the $i$ th class. As the image samples of the same class usually share some common 
patterns, the sample $y$ is more likely to be represented by the training samples from the same class as it. In other words, if $y$ belongs to a certain class $k$, the following inequality $\sum_{j} \alpha_{k, j}>\sum_{j} \alpha_{i, j}, \forall i=1,2, \cdots, c$, and $i \neq k$ should be valid. Then we can treat the ratio below as the classification criterion for $y$ :

$$
L(y)=\arg \max _{i} \frac{\sum_{j} \alpha_{i, j}}{\sum_{i, j} \alpha_{i, j}}
$$

This term $\sum_{j} \alpha_{i, j} / \sum_{i, j} \alpha_{i, j}$ will be adopted as the probability of belonging to class $i$ for $y$. Meanwhile, by picking out the coefficients related to different concepts and adding them up separately, we can obtain the forward confidences $C_{f}=\left\{\sum_{j} \alpha_{i, j} / \sum_{i, j} \alpha_{i, j}, i=1, \cdots, c\right\}$ for the fusion procedure later.

As the equation $X u u^{T} \alpha=X \alpha$ with $u$ in Eqn.(2) as a scalar is satisfied and $\alpha$ is used to measure the similarities between testing images and training ones directly, we should normalize each column atom $x_{i, j}$ of $X$ to a unit vector for fair in the same level.

\subsection{Backward Sparse Representation}

From the opposite direction, under the semi-supervised assumption, we can treat the whole testing data $Y \in R^{d \times N}$ as the dictionary to represent the samples in $X$ and bridge the correlations between training data and testing data from a different viewpoint. Then similar to Eqn.(1), any training image $x_{i, j}$ in Eqn.(3) can be linearly represented as follows

$$
x_{i, j}=Y b_{i, j}+e
$$

where $b_{i, j}=\left[\beta_{i, j}^{1}, \cdots, \beta_{i, j}^{N}\right]^{T} \in R^{N}$ are the reconstruction coefficients related to $Y$ for the sample $x_{i, j}$. Similar to Eqn.(2), minimizing the following objective function can obtain the corresponding $b_{i, j}$,

$$
\min _{b_{i, j}}\left\|x_{i, j}-Y b_{i, j}\right\|^{2}+\lambda\left\|b_{i, j}\right\|_{1}
$$

After obtaining all the $b_{i, j}$ for the whole training data $X$, we will get a 
coefficient matrix $B$ as below,

$$
\begin{aligned}
B & =\left[b_{1,1}, \cdots, b_{1, m_{1}}, \cdots, b_{c, 1}, \cdots, b_{c, m_{c}}\right] \\
& =\left[\begin{array}{ccccccc}
\beta_{1,1}^{1} & \cdots & \beta_{1, m_{1}}^{1} & \cdots & \beta_{c, 1}^{1} & \cdots & \beta_{c, m_{c}}^{1} \\
\vdots & \vdots & \vdots & \vdots & \vdots & \vdots & \vdots \\
\beta_{1,1}^{N} & \cdots & \beta_{1, m_{1}}^{N} & \cdots & \beta_{c, 1}^{N} & \cdots & \beta_{c, m_{c}}^{N}
\end{array}\right]
\end{aligned}
$$

The coefficients $\beta$ associated with each testing image $y$ can be achieved by picking out corresponding rows in Eqn.(7).

$$
\beta=\left[\beta_{1,1}, \cdots, \beta_{1, m_{1}}, \cdots, \beta_{c, 1}, \cdots, \beta_{c, m_{c}}\right]
$$

Similar to fSR, if $y$ is from class $k$, training samples from the same class $k$ are more likely to own bigger weights. Of course, it will be affected by noise and concept correlations. However, if all the testing images as the dictionary can represent training images well enough, the weights in $\beta$ associated with class $i(i \neq k)$ should still be small. Thus the following inequality $\sum_{j} \beta_{k, j}>$ $\sum_{j} \beta_{i, j}, \forall i=1,2, \cdots, c$, and $i \neq k$ should also be valid. Then the following ratio can be treated as the classification criterion for each testing image $y$ :

$$
L(y)=\arg \max _{i} \frac{\sum_{j} \beta_{i, j}}{\sum_{i, j} \beta_{i, j}}
$$

225 Accordingly, the term $\sum_{j} \beta_{i, j} / \sum_{i, j} \beta_{i, j}$ will also be used to show the degree of belonging to class $i$ for $y$. By picking out the coefficients corresponding to different concepts and adding them up separately, the backward confidences $C_{b}=\left\{\sum_{j} \beta_{i, j} / \sum_{i, j} \beta_{i, j}, i=1, \cdots, c\right\}$ for the fusion procedure later can be obtained. The normalization operation for all the images in testing data $Y$ should be conducted to keep them fair in the same level.

\subsection{Fusion of Forward and Backward Sparse Representation}

According to the theory of co-training, if these two opposite sparse representations are conditionally independent and sufficient, the fusion of them will provide complementary information and improve classification performance. Then a simple but effective way, namely weighted-averaging fusion, can be adopted 
to combine the output scores of both SRs. As there may exist negative values in sparse coefficients, a max-min normalization will be performed to keep all the values among $[0,1]$. Then the fusion expression showing the probability of belonging to class $k$ for the testing sample $y$ is as follows:

$$
\begin{aligned}
\eta_{k} & =w * C_{f}(k)+(1-w) * C_{b}(k) \\
& =w * \frac{\sum_{j} \alpha_{k, j}}{\sum_{i, j} \alpha_{i, j}}+(1-w) * \frac{\sum_{j} \beta_{k, j}}{\sum_{i, j} \beta_{i, j}}
\end{aligned}
$$

$w$ is a tradeoff parameter controlling the importance of different SRs. We apply $\ell_{1}$ normalization, which divides $\alpha_{k, j}$ and $\beta_{k, j}$ by the sum of all the elements in the vectors respectively, to make them mixed in the same order of magnitude.

Now $\eta_{k}$ can show the relevance between training images in different classes and the testing ones in $Y$. Finally the following classification criterion will be adopted to decide the label for $y$ :

$$
L(y)=\max _{k} \eta_{k}
$$

Taking the annotation labels of samples from the same class and supplementing them with co-training technique, an iterative procedure can be adopted to improve the final results.

\section{Image Set Classification via Cooperative Sparse Representation}

The details of the extended CoSR model for image set classification will be provided in this section. At first, a problem description for image set classification is defined as below.

Problem Description. During training, for $c$ classes of a training data, we are given $c$ image sets $X=\left[X_{1}, X_{2}, \ldots X_{c}\right] \in R^{d \times m}$ and their corresponding class labels $l_{i} \in\{1,2, \ldots c\}$. An image set $X_{i}$ contains all $m_{i}$ training images belonging to class $i$ and $X_{i}=\left\{x_{i, t} \mid l_{i, t}=i, t=1,2, \cdots, m_{i}\right\}$. For concepts with multiple image sets, we combine images from all the sets into a single set. During classification, we are given a query image set $Q=\left[q^{1}, q^{2}, \ldots, q^{N_{q}}\right] \in R^{d \times N_{q}}$, and the task is to find the class label $L_{Q}$ for the whole set . 


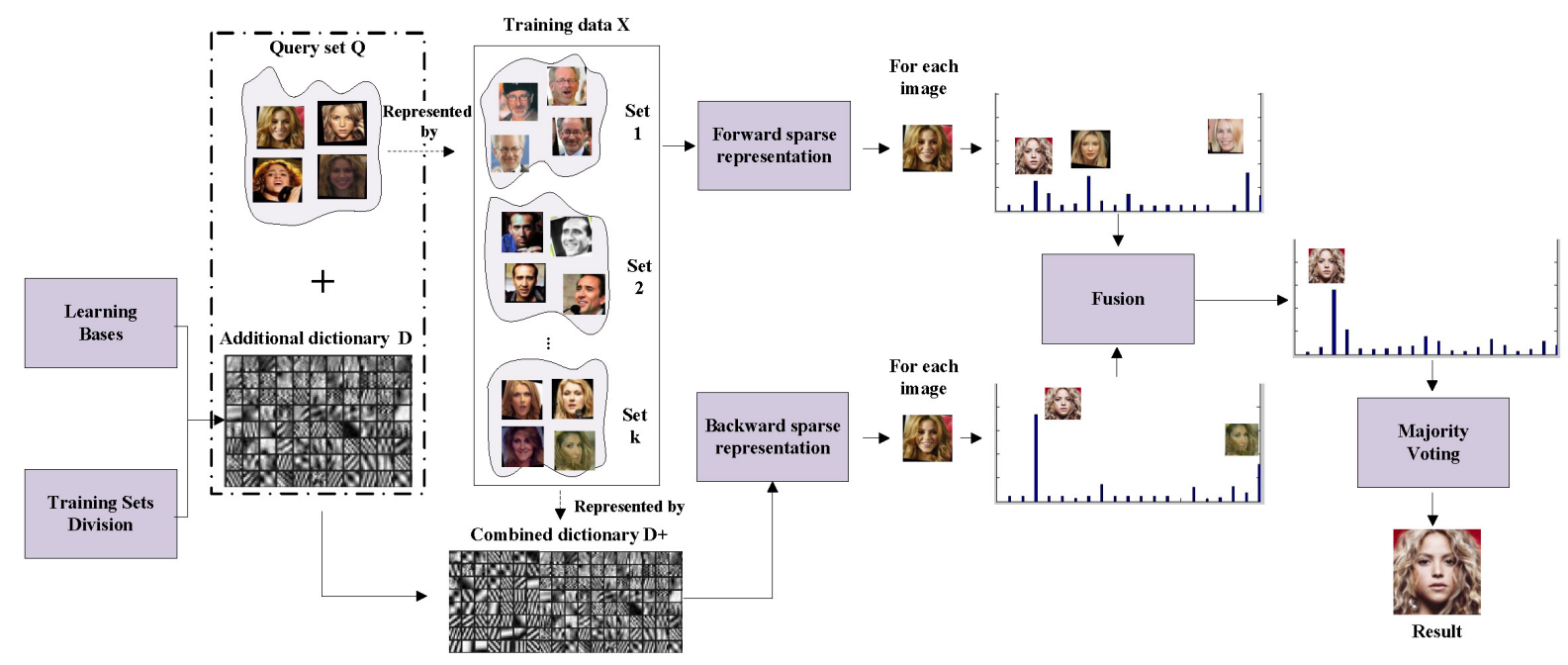

Figure 1: The process of Cooperative Sparse Representation model for image set classification. There are two main components - forward sparse representation (fSR) and backward sparse representation (bSR). For fSR, each sample in query set $\mathrm{Q}$ is represented by training data $X$ to obtain forward confidences. For bSR, it is not applicable to represent samples in $X$ with $Q$ directly. So two schemes, namely 'Learning Bases' and 'Training Sets Division', are taken to construct additional dictionary $D$. Backward confidences are achieved by representing $X$ with the combination $\left(D^{+}\right)$of $D$ and $Q$. Finally, majority voting is applied to the fusion confidences of fSR and bSR for all the query samples to get the final classification result.

\subsection{From Image Annotation to Image Set Classification}

The whole flow chart of the cooperative sparse representation model for image set classification is shown in Fig.(1). It is also categorized into three parts, namely forward sparse representation with training sets $X$ to represent query set $Q$, backward sparse representation with the combination of $Q$ and learned additional dictionary $D$ to represent training sets $X$ and the fusion of the above two SRs' decisions.

For image set classification, the procedure of fSR is similar to that of image annotation, where the label of each sample $q^{s}$ in $Q$ can be decided through Eqn. (9). However, for the procedure of bSR, it is quite different. The principle of bSR is to use query set as the dictionary to represent training images. However,

the samples of $Q$ are all from the same concept, which are not diverse and 
discriminant enough to represent different training images well. And it usually does not comply with the rules of over-complete for sparse representation. So a set of additional bases $D=\left[d_{1}, d_{2}, \cdots, d_{n}\right] \in R^{d \times n}$ taking possessions of visual features of other concepts should be provided at first.

With $D$ and query set $Q$, Eqn.(5) will be modified as follows:

$$
\begin{aligned}
x_{i, j} & =\left[\begin{array}{ll}
Q & D
\end{array}\right]\left[\begin{array}{c}
b_{i, j} \\
\hat{b}
\end{array}\right]+e \\
& =D^{+} b_{i, j}^{+}+e
\end{aligned}
$$

where $b_{i, j}=\left[\beta_{i, j}^{1}, \cdots, \beta_{i, j}^{N_{q}}\right]^{T} \in R^{N_{q}}$ are the reconstruction coefficients related to $Q$ for the sample $x_{i, j}$. Similarly, the objective function of Eqn. (6) will be redefined as below to obtain the corresponding $b_{i, j}$,

$$
\min _{b_{i, j}^{+}}\left\|x_{i, j}-D^{+} b_{i, j}^{+}\right\|^{2}+\lambda\left\|b_{i, j}^{+}\right\|_{1}
$$

Having obtained the confidence matrices from fSR and bSR, the fusion of these two SRs will be conducted via Eqn.(10). After having determined the labels for all the samples in $Q$, the query label $L_{Q}$ can be obtained via Majority Voting scheme as follows:

$$
\begin{gathered}
L_{Q}=\arg \max _{k} \sum_{s} \delta_{k}\left(L\left(q^{s}\right)\right), \text { where } \\
\delta_{k}\left(L\left(q^{s}\right)\right)= \begin{cases}1, & L\left(q^{s}\right)=k \\
0, & \text { otherwise }\end{cases}
\end{gathered}
$$

\subsection{The Procedure of bSR for Image Set Classification}

As described above, for bSR we only have the query set $Q$ itself available and it is not enough to accomplish the procedure of bSR with $Q$ as the dictionary directly. So some additional bases should be learned to complement with $Q$. To reduce the reconstruction residuals and improve the performance of bSR, we take two methods, namely 'Learning Bases' and 'Training Sets Division', to construct the needed bases. 


\subsubsection{Learning Bases for bSR}

Apparently, some random bases can be adopted to help us perform bSR. However, according to the theory of dictionary learning, these bases are usually not discriminant enough to separate images of different visual concepts well. Therefore, to improve the performance of bSR, the learning of these bases is of necessity. In this paper, the optimization objective function is as follows,

$$
\begin{gathered}
\sum_{i=1}^{c} \sum_{j=1}^{m_{i}} \min _{D, S}\left\|x_{i, j}-D^{+} b_{i, j}^{+}\right\|^{2}+\lambda\left\|b_{i, j}^{+}\right\|_{1} \\
\text { subject to }\left\|d_{l}\right\|^{2} \leq 1, \forall l=1, \cdots, n
\end{gathered}
$$

where $S$ is the sparse coefficient matrix associated with $X, D^{+}=\left[\begin{array}{ll}Q & D\end{array}\right] \in$ $R^{d \times\left(N_{q}+n\right)}$. This is the standard optimization objective function in dictionary learning, which is convex when optimizing $D$ with $S$ fixed and vice versa. Suppose that we have already obtained the initial sparse coefficient matrix $B$ as Eqn. (7) shows, the optimization of Eqn. (15) turns to the problem of learning $D$ with fixed $B$ via the following minimization function:

$$
\begin{gathered}
\min _{D}\left\|X-[Q \quad D]\left[\begin{array}{c}
B \\
S
\end{array}\right]\right\|^{2} \\
\text { subject to }\left\|d_{l}\right\|^{2} \leq 1, \forall l=1, \cdots, n
\end{gathered}
$$

Instead of solving Eqn. (16) via gradient descent algorithm which is a repeating iterative projection optimization with varying sparse coefficient values, we use the Lagrange dual algorithm in 30] to learn bases $D$ for efficiency. Transferring Eqn.16) into the following Lagrangian:

$$
\begin{aligned}
& \mathcal{L}(D, \vec{\theta})=\sum_{l=1}^{n} \theta_{l}\left(\left\|d_{l}\right\|^{2}-1\right)+ \\
& \operatorname{trace}\left(\left(X-\left[Q\left[\begin{array}{ll}
Q & D
\end{array}\left[\begin{array}{c}
B \\
S
\end{array}\right]\right)\right)^{T}\left(X-[Q \quad D]\left[\begin{array}{l}
B \\
S
\end{array}\right]\right)\right)\right.
\end{aligned}
$$


where each $\theta_{l} \geq 0$ is a dual variable and $\vec{\theta}=\left[\theta_{1}, \cdots, \theta_{n}\right]$. Then the following Lagrange dual can be obtained by minimizing over $D$ analytically,

$$
\begin{aligned}
\mathcal{D}(\vec{\theta})= & \min _{D} \mathcal{L}(D, \vec{\theta}) \\
= & \operatorname{trace}\left((X-Q B)^{T}(X-Q B)-\right. \\
& \left.(X-Q B) S^{T}\left(S S^{T}+\Theta\right)^{-1}\left((X-Q B) S^{T}\right)^{T}-\Theta\right)
\end{aligned}
$$

where $\Theta=\operatorname{diag}(\vec{\theta})$. The corresponding partial gradient and Hessian for $\mathcal{D}(\vec{\theta})$ over $\theta_{l}$ are computed as follows,

$$
\begin{gathered}
\frac{\partial \mathcal{D}(\vec{\theta})}{\partial \theta_{l}}=\left\|(X-Q B) S^{T}\left(S S^{T}+\Theta\right)^{-1} e_{l}\right\|^{2}-1 \\
\frac{\partial^{2} \mathcal{D}(\vec{\theta})}{\partial \theta_{l_{1}} \theta_{l_{2}}}=-2\left(\left(S S^{T}+\Theta\right)^{-1}\left((X-Q B) S^{T}\right)^{T}\right. \\
\left.(X-Q B) S^{T}\left(S S^{T}+\Theta\right)^{-1}\right)_{l_{1}, l_{2}}\left(\left(S S^{T}+\Theta\right)^{-1}\right)_{l_{1}, l_{2}}
\end{gathered}
$$

where $e_{l} \in R^{n}$ is the $l$-th unit vector. With Newton's method or conjugate gradient, the optimization of Eqn.17 can be achieved. By maximizing $\mathcal{D}(\vec{\theta})$, we will get the optimal dictionary $D_{\text {opt }}$ as follows,

$$
D_{o p t}^{T}=\left(S S^{T}+\Theta\right)^{-1} S X^{T}-\left(S S^{T}+\Theta\right)^{-1} S B^{T} Q^{T}
$$

As there are much fewer variables to optimize, this dual algorithm is more efficient than the primal gradient descent algorithm. And with the optimal $D_{\text {opt }}$, the final sparse coefficient matrix $B$ for bSR will also be obtained.

\subsubsection{Training Sets Division for bSR}

Considering that usually there are tens or even hundreds of images in each set for image set classification task and the samples in the same set have smaller visual variants, a subset of samples for each set still own a relatively high discriminant ability. Meanwhile, natural images are easier to obtain and contain abundant information for recognizing different concepts compared with artificial learned dictionary. They are the basic assumptions of the second proposed scheme and guide us to make use of existing training samples to construct additional bases $D$. 


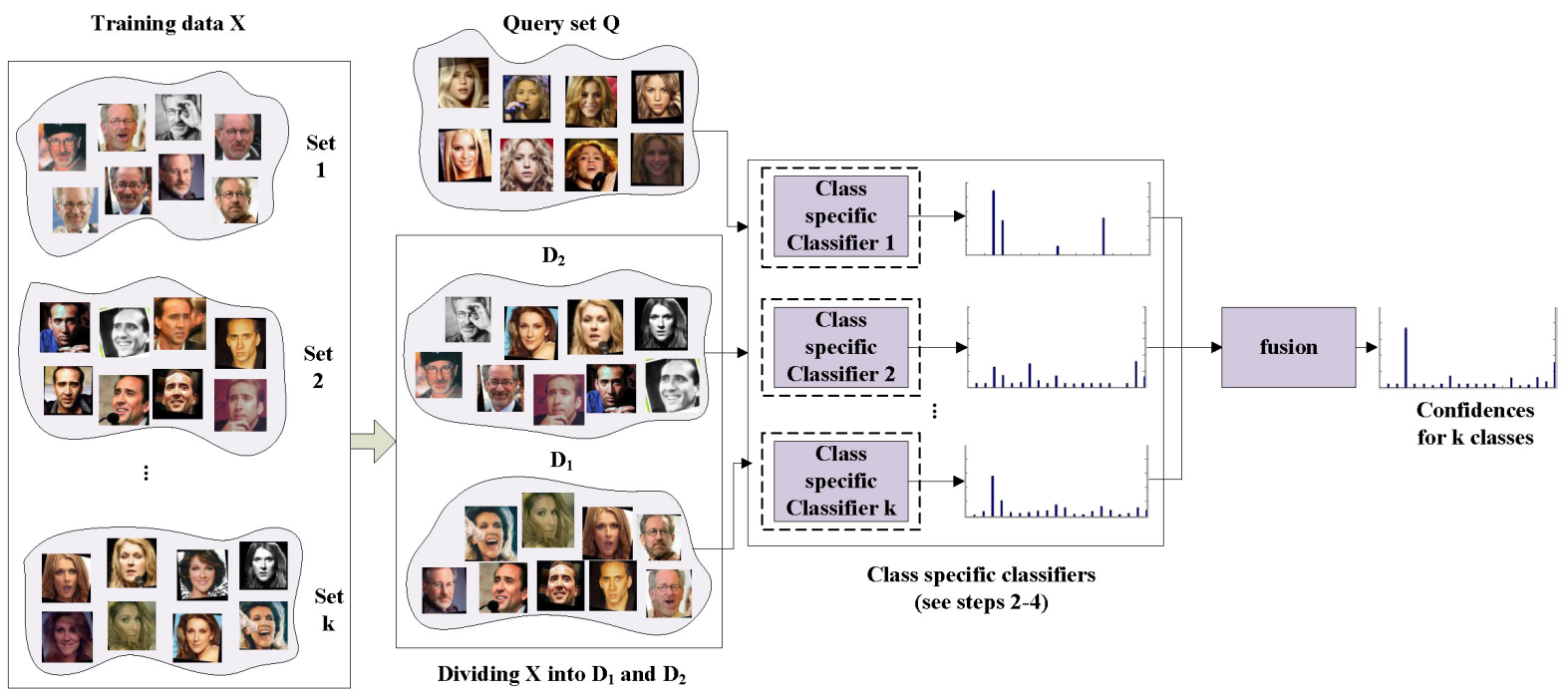

(a) Block diagram of the proposed 'Training Sets Division' scheme for bSR

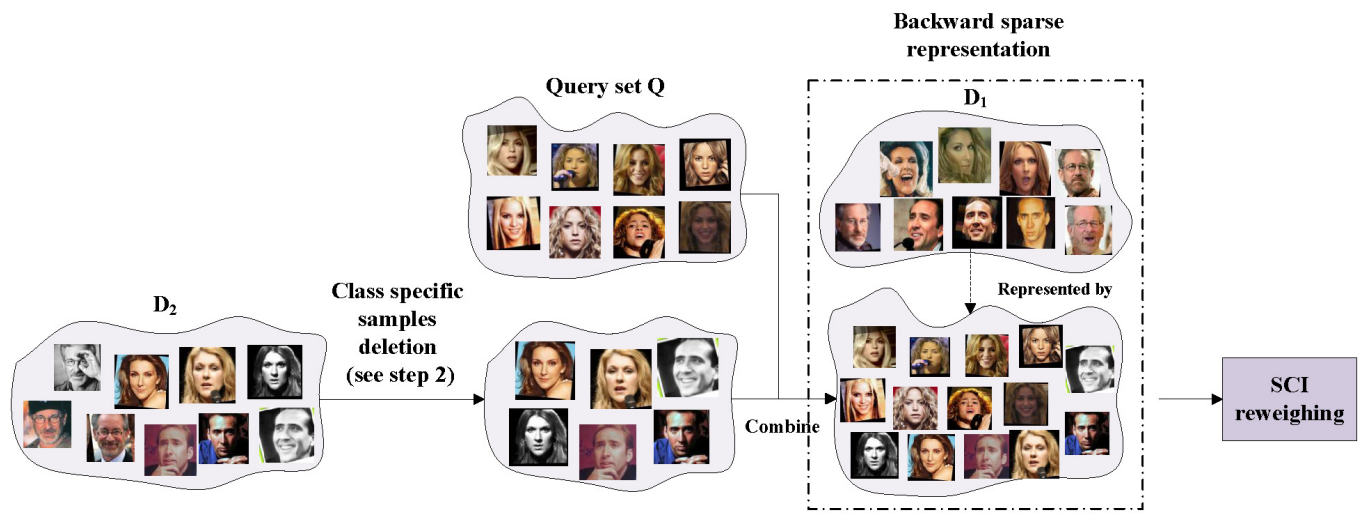

(b) The details for the dotted box of each class specific classifier in (a)

Figure 2: The details of the proposed 'Training Sets Division' scheme for bSR. Firstly, training data $X$ is divided into two sets $\mathcal{D}_{1}$ and $\mathcal{D}_{2}$. Based on query set $Q$ and them, $c$ class specific classifiers are trained. And the final confidences for $c$ classes are obtained by fusing the outputs of all the classifiers. For each classifier $i$, class specific samples deletion is conducted to $\mathcal{D}_{2}$ and modified $\mathcal{D}_{2}$ is obtained by combining it with query set $Q$ after that. By representing $\mathcal{D}_{1}$ with modified $\mathcal{D}_{2}$ via backward sparse representation and reweighing obtained coefficients with sparsity concentration index (SCI), a class specific classifier is trained. 
trated in Fig.(2) and its pseudo code is summarised in Alg.(1). The details for every step are presented below.

Step 1. All the training sets are merged into one set $\mathcal{D}=\left\{X_{1}, X_{2}, \cdots, X_{c}\right\}$. Then $\mathcal{D}$ will be divided into two subsets: $\mathcal{D}_{1}$ and $\mathcal{D}_{2}$. Let $D_{1 i}$ be a sampled subset of $X_{i}$ with a set size of $N_{D_{1 i}}$, where $N_{D_{1 i}}=\gamma * m_{i}$ and $\gamma$ is a parameter to decide how many percents of the samples in $X_{i}$ are contained in $D_{1 i}$. Next, the union $\left\{\bigcup_{i} \mathcal{D}_{1 i}, i=1,2, \cdots, c\right\}$ forms $\mathcal{D}_{1}$ and $\mathcal{D}_{2}$ is achieved by $\mathcal{D}_{2}=\mathcal{D} \backslash$ $\mathcal{D}_{1},\left(\mathcal{D}_{2}=\bigcup_{i} \mathcal{D}_{1 i}, i=1,2, \cdots, c\right)$. The class labels of the images in $\mathcal{D}_{1}$ and $\mathcal{D}_{2}$ are stored in sets $\mathbf{y}_{\mathcal{D}_{1}}=\left\{y^{(t)} \in[1,2, \cdots, c], t=1,2, \ldots, N_{\mathcal{D}_{1}}\right\}$ and $\mathbf{y}_{\mathcal{D}_{2}}=\left\{y^{(t)} \in\right.$ $\left.[1,2, \cdots, c], t=1,2, \ldots, N_{\mathcal{D}_{2}}\right\}$ respectively.

To obtain $\mathcal{D}_{1}$ and $\mathcal{D}_{2}$, we adopt the Hierarchical Divisive Clustering (HDC) in [5] to cluster each set into several subsets named maximal linear patches (MLPs). This algorithm treats each set as a manifold and takes the deviation between Euclidean distances and geodesic distances to measure nonlinearity degrees of local patches. More details can be found in [5]. Then we divide each MLP into two parts according to $\gamma$ to form $\mathcal{D}_{1}$ and $\mathcal{D}_{2} \cdot($ remark 1$)$

Step 2. For each class $i$, we remove all the samples of this class from $\mathcal{D}_{2}$, namely $\mathcal{D}_{2}^{-}=\mathcal{D}_{2}-\mathcal{D}_{2 i}$, and this operation is called Class Specific Samples Deletion. Then $Q$ will be combined with $\mathcal{D}_{2}^{-}$to form $\mathcal{D}_{2}^{+}$, as $\mathcal{D}_{2}^{+}=\mathcal{D}_{2}^{-} \cup Q$ (remark 2).

Step 3. With $\mathcal{D}_{2}^{+}$, we can use Eqn.13 to calculate corresponding sparse coefficients $b_{j}$ for each $x_{j}$ in $\mathcal{D}_{1}$ and concept sparse matrix $B_{j}$ will be formed with $\bigcup_{j} b_{j}$ (remark 3 ). Here each $B_{i}$ can be regarded as a classifier and processed individually. Then this step can also be considered as a component of the construction for Class Specific Classifiers. Final decisions will be made by taking the output scores from all the classifiers into consideration.

Step 4. As there are c classifiers and some of them have poor qualities due to the greedy scheme above, a quality measure can be introduced for bias. To decide whether a sparse representation owns a good quality or not, in this 


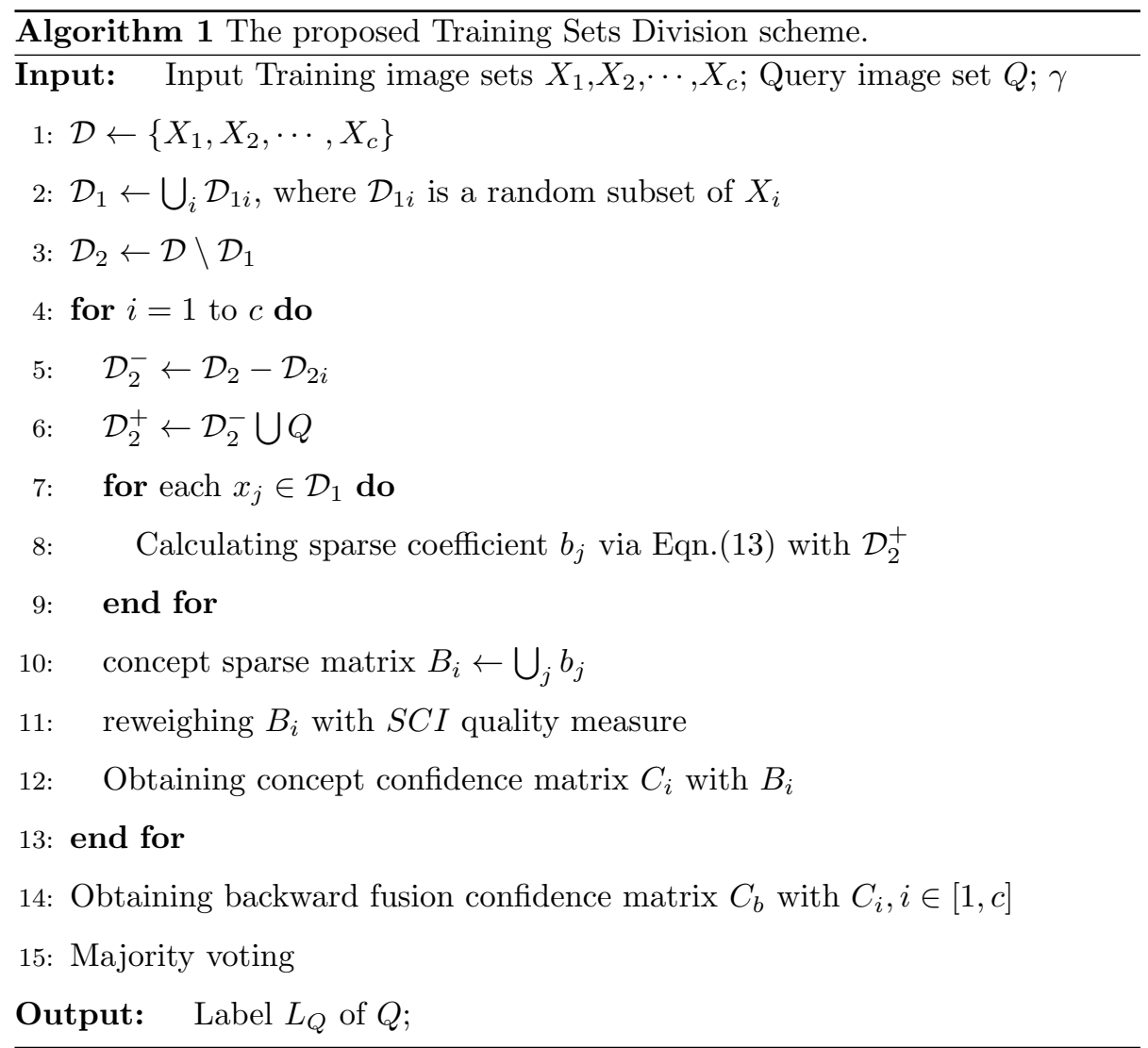

paper, sparsity concentration index (SCI) is calculated. Given a coefficient vector $\gamma \in R^{d}$, the SCI is given as

$$
S C I(\gamma)=\frac{\frac{c * \max _{j \in\{1, \ldots, c\}}\left\|\delta_{j}(\gamma)\right\|_{1}}{\|\gamma\|_{1}}-1}{c-1}
$$

where $\delta_{j}$ is an indicator function with the coefficients related to the $j$-th class unchanged and the others set to zero (remark 4). By multiplying each column of $B_{i}$ (the representation for a sample in $\mathcal{D}_{1}$ ) with its SCI value, the obtained confidence matrices will be more accurate.

Step 5. Given all the $c$ sparse coefficient matrices, the next job is to fuse them to get a final confidence matrix indicating the probability of belonging to different classes for each sample in $Q$. With Eqn.(7) and Eqn.(8), we can transfer each $B_{i}$ to a concept confidence matrix $C_{i}$ by picking out the coefficients related 
to different concepts and adding them up separately $\left(C_{i} \in R^{N_{q} \times c}\right)$. Here an $\ell_{l}$-norm should be applied to each row in $C_{i}$ to make the summed probabilities equal to 1 .

Step 6. For simplicity, final backward confidence matrix $C_{b}$ is formed by 325 adding all the single concept confidence matrices up directly. Some reweighing schemes can be adopted to weigh the importance of different classifiers for better performance. To decide $L_{Q}$, Majority Voting scheme will be applied to combine the information from all the query samples.

Remarks:

1) The reasons why we take this scheme come in two folds. On one hand, as the extracted MLPs are balanced and the samples in the same MLP are visually similar to each other, which is proved in [5], the new training data $\mathcal{D}_{1}$ and dictionary $\mathcal{D}_{2}$ will be more balanced and diverse. On the other hand, in order to improve efficiency, we need to pick out the most relevant $k$ concepts and compress each set into several mean samples as described in Sec.(4.3). As representing each set with a mean image will produce very a poor result for bSR, we applied these schemes to the extracted MLPs as alternative ways to obtain tens of representative samples each concept for the given 340 query images.

2) In theory, after the division of $\mathcal{D}$, we can consider $\mathcal{D}_{1}$ and $\mathcal{D}_{2}$ as the new training data and additional dictionary respectively to accomplish the procedure of bSR. However, as the samples from the same set in these two subsets usually own more similarities, they are more likely to be represented by each other even that query set $Q$ has the same class label as them. So we must avoid this kind of situation. In this paper, we propose to use a greedy method to solve this problem.

3) For the correct concept $k$, its samples in $\mathcal{D}_{1}$ tend to be represented by the images in $\mathcal{D}_{2}^{+}$(from the same set) if $\mathcal{D}_{2 k}$ is not removed, while by the images 350 in $Q$ if $\mathcal{D}_{2 k}$ is removed. In the meantime, for the incorrect concepts $i(i \neq k)$, their samples in $\mathcal{D}_{1}$ will be related to the images in $\mathcal{D}_{2}^{+}$(from the same set) if 
$\mathcal{D}_{2 i}$ is not removed, while scatteredly represented by the samples from all the concepts if $\mathcal{D}_{2 i}$ is removed as there are not any images from $i$ left. So it is more likely for query set $Q$ to be correctly classified.

4) The closeness to 1 stands for the case where the test sample can be well represented by the samples of a single class, hence the representations are of high quality. On the contrary, samples with SCI close to 0 are not similar to any of the classes, and hence their representations are of poor quality.

\subsection{Computational Complexity Analysis}

is made on the proposed model and accordingly several promotion strategies are provided to improve the efficiency.

The most time-consuming part of the model falls in bSR, as fS$R$ is a traditional sparse representation which just calculates sparse k-means method to cluster the samples of each extracted MLP in Sec.(4.2.2) into smaller subsets, as there may still exist tens or even hundreds of images in the MLP. Then the mean image of each subset is calculated and normalized to represent the whole subset. By doing

2)KNN: To reduce the number of classifiers in 'TSD', pair-wise 
local model distances are computed between the centers of extracted MLPs using Euclidean distance measurement. Then the similarities of training sets and query set are obtained by matching their closest pairs of local models. Based on the similarities, $k$ most similar sets are selected to construct the corresponding classifiers, which reduces the number of classifiers from $c$ to $k$.

With these two promotion schemes, the efficiency of the proposed model is improved while the performance is not affected so much. Also parallel techniques and the scheme in 31 can be adopted to improve efficiency further.

\subsection{Guidelines for the Selection of Different Schemes}

In this subsection, some guidelines for the proposed model will be provided, which alternatively gives a comparison between these two schemes for bSR.

1) As the discriminant bases for ' $\mathrm{Co}+\mathrm{LB}$ ' need to be trained at first, which may encounter the problem of overfitting for lack of training samples, this scheme is more suitable for the tasks with abundant samples. For 'Co+TSD', there should be a balance between the size of training data and the dimension of features to reach the assumption of over-complete.

2)As it is easier to obtain artificial discriminant dictionary for facial images (they do not have so many fine structures than objects and scenes), 'Co+LB' is likely to produce better results on face recognition task. The additional bases for 'Co+TSD' are picked from natural images, which makes 'Co+TSD' suitable for various applications.

3) 'Co+TSD' needs to calculate sparse codes for more samples than 'Co+LB' even both the schemes in Sec. (4.3) are adopted. It indicates that 'Co+TSD' is more suitable for the tasks paying more attention to accuracy than speed. 


\section{Experimental Results and Discussions}

We evaluate and compare the performance of the proposed method with existing methods on several benchmark datasets including Honda/UCSD dataset [32, CMU Mobo dataset [33], ETH-80 dataset [34] and Scenes 15 dataset 35]. They represent different sub-tasks of image set classification, namely face identification, object categorization and scene classification, and have their own characteristics. At first, experimental settings are presented. Then the detailed description of each dataset and the performance evaluation of all the method$\mathrm{s}$ will be shown. And the comparisons among different versions of the CoSR model, namely 'CoSR with Learning Bases (Co+LB)' and 'CoSR with Training Sets Division (Co+TSD)', are also exhibited. Finally, an ablative analysis for the method is provided.

\subsection{Experimental Settings}

For all the datasets, the colored images are converted to gray scale levels. The face frames in the videos of Honda/UCSD and Mobo datasets are automatically detected with Viola and Jones face detection algorithm [36. The face region is cropped after a successful detection. Then the cropped gray scale images are resized to $20 \times 20,40 \times 40$ for Honda/UCSD and Mobo datasets respectively. For object dataset (ETH-80), the original $128 \times 128$ cropped images are resized to $32 \times 32$. And the size of the images in Scenes 15 is kept as $300 * 200$. Histogram equalization is conducted on all the images to minimize illumination variations. At the same time, the features used for Honda/UCSD and ETH- 80 are raw pixel values. For Mobo dataset, the concatenated LBP histograms from $4 \times 4$ non-overlapping uniformly spaced rectangular blocks in features from [17] are used to model each set.

\subsubsection{Settings of Our Method}

Here we provide the settings of our method. For all the datasets except for Scenes 15 , we reduce the dimension of the features to 100 via PCA. To calculate 
sparse representation coefficients, we use the homotopy algorithm showing to possess a relatively good performance and a low time complexity provided on the webpage (http://users.ece.gatech.edu/\%7Esasif/homotopy/). We set the tradeoff parameter $\lambda$ to 0.01 and the corresponding convergence tolerance to 0.001 for all the datasets. For 'Learning Bases' scheme, the size $n$ of additional dictionary is ranging between 1500 and 600 . The problem of overfitting may be encountered when we try to obtain a large dictionary with a quite small training set, and it can be solved by deforming images to obtain more training instances [37]. Meanwhile, for 'Training Sets Division' scheme, the ratio $\gamma$ between $N_{\mathcal{D}_{1}}$ and $N_{\mathcal{D}}$ takes the value from 0.5 to 0.9 . Also the parameters for 'HDC' strategy are set the same as those of [5] and the parameter $k$ in Sec. (4.3) is set to 3 for all the datasets. When it turns to the fusion procedure, the weighing parameter $w$ is located among $[0,1]$ and optimized for the best performance according to different datasets via cross-validation. For datasets with a few samples, the deformed images produced by [37] can be used as validation data to tune the parameter. Here a value of 1 means conducting fSR only while a value of 0 means experimenting with bSR only.

\subsubsection{Settings of Compared Methods}

Comparisons between a number of recently proposed state of the art image set classification methods and our proposed method are conducted. The compared methods include Mutual Subspace Method (MSM) [1], the Linear version of the Affine Hull-based Image Set Distance (AHISD) [6], the Convex Hull-based Image Set Distance (CHISD) 6], Discriminant Canonical Correlation Analysis (DCC) [3, Manifold-to-Manifold Distance (MMD) 4], Manifold Discriminant Analysis (MDA) [5], Sparse Approximated Nearest Points (SANP) [8], Regularized Nearest Points (RNP) [38], Covariance Discriminant Learning (CDL) 14, Mean Sequence Sparse Representation Classification (MSSRC) [13] and Set to Set Distance Metric Learning (SSDML) [39]. For all the methods, their parameters are tuned for the best performance. Specifically, for MSM, 
for AHISD. For CHISD, the error penalty term $(\mathrm{C}=100)$ in SVM is kept as that in [6]. For DCC, the dimensions of the embedding space are set to 100. The retained dimensions for a subspace after dimension reduction are set to 10 (90\% of the energy is preserved) and the corresponding 10 maximum canonical 475 correlations are used to compute set-to-set similarity. To construct the within class sets for datasets with one training set per class (Honda/UCSD, Mobo and Scenes 15), the training set is randomly divided into two subsets as that in [3]. The parameters for MMD and MDA are kept the same as those in [4] and [5] respectively. For geodesic distance, the number of connected nearest neighbors takes the value of 12 or the smallest set size of the dataset. The ratio between Euclidean distance and geodesic distance is optimized for all the datasets. For MMD, its distance is computed based on maximum canonical correlation. For SANP, we adopt the same weight parameters for convex optimization as $[8]$. No parameters are required for CDL. For RNP [38, 90\% of the energy is preserved by PCA and the weight parameters are kept the same as those in 38. No parameter configurations are required for MSSRC and SSDML.

\subsection{Results and Analysis}

\subsubsection{Honda/UCSD Dataset}

The Honda/UCSD dataset 32 is formed by 59 video sequences of 20 different subjects. Each video sequence contains a number of frames varying from 12 to 645. Following the standard evaluation configuration provided in [32], each video is regarded as an image set. Then 20 video sequences (one for each subject) are used for training while the remaining 39 ones are kept as query sets. To achieve a consistency in the results, the experiments are conducted for 10 times with

To show the robustness of different methods, their performance is evaluated by reducing the size of the training and query sets as 38 . That is to say, an upper limit is added to the total number of images in the training and query sets respectively. Here we make experiments by considering $N_{\text {train }}=$ 


\subsection{2. $C M U$ Mobo Dataset}

Originally, the Mobo (Motion of Body) dataset 33] was constructed for the pose identification of human body. This dataset consists of 96 sequences of 
Table 1: Performance on Honda/UCSD dataset

\begin{tabular}{cccccccc}
\hline Methods & All-All & $100-100$ & $50-50$ & $50-25$ & $50-15$ & $25-50$ & All-1 \\
\hline MSM [11] & $91.3 \pm 1.9$ & $85.6 \pm 4.4$ & $83.1 \pm 1.7$ & $82.3 \pm 4.3$ & $80.7 \pm 5.0$ & $79.2 \pm 5.0$ & $57.7 \pm 12.3$ \\
DCC [3] & $92.6 \pm 2.3$ & $89.3 \pm 2.5$ & $82.1 \pm 3.3$ & $80.8 \pm 8.8$ & $80.2 \pm 3.4$ & $78.4 \pm 2.7$ & $3.8 \pm 3.0$ \\
MMD [4] & $92.1 \pm 2.3$ & $85.6 \pm 2.2$ & $83.1 \pm 4.5$ & $82.4 \pm 5.2$ & $80.4 \pm 3.6$ & $81.2 \pm 3.8$ & - \\
MDA [5] & $94.4 \pm 3.4$ & $91.8 \pm 1.6$ & $85.6 \pm 5.8$ & $85.0 \pm 4.0$ & $83.7 \pm 5.8$ & $84.7 \pm 4.5$ & - \\
AHISD [6] & $88.6 \pm 2.8$ & $92.8 \pm 2.2$ & $94.9 \pm 1.8$ & $93.9 \pm 2.9$ & $93.9 \pm 2.3$ & $92.6 \pm 2.3$ & $76.7 \pm 7.7$ \\
CHISD [6] & $90.3 \pm 1.2$ & $92.3 \pm 1.8$ & $92.3 \pm 0.0$ & $93.3 \pm 2.9$ & $93.3 \pm 2.9$ & $91.5 \pm 2.5$ & $79.0 \pm 6.4$ \\
SANP [8] & $93.3 \pm 2.9$ & $94.9 \pm 2.6$ & $94.9 \pm 2.6$ & $94.3 \pm 3.8$ & $92.8 \pm 2.2$ & $91.3 \pm 2.9$ & $54.9 \pm 11.4$ \\
CDL [14] & $\mathbf{9 6 . 4} \pm \mathbf{2 . 9}$ & $95.4 \pm 2.2$ & $91.3 \pm 3.4$ & $85.1 \pm 7.1$ & $71.3 \pm 8.6$ & $80.0 \pm 3.3$ & $5.1 \pm 0.0$ \\
MSSRC [13] & $88.2 \pm 3.9$ & $89.7 \pm 2.6$ & $88.7 \pm 2.3$ & $90.8 \pm 3.9$ & $88.2 \pm 3.9$ & $89.2 \pm 3.3$ & $79.0 \pm 6.9$ \\
SSDML [39] & $93.3 \pm 3.9$ & $95.4 \pm 2.8$ & $95.9 \pm 4.7$ & $92.8 \pm 5.3$ & $92.8 \pm 5.3$ & $92.8 \pm 4.6$ & $68.7 \pm 3.3$ \\
RNP [38] & $95.9 \pm 2.2$ & $92.3 \pm 3.2$ & $90.2 \pm 3.3$ & $89.3 \pm 6.6$ & $85.4 \pm 2.0$ & $84.8 \pm 2.7$ & $40.0 \pm 7.4$ \\
\hline CoSR+LB & $\mathbf{9 6 . 9} \pm \mathbf{2 . 2}$ & $\mathbf{9 7 . 4} \pm \mathbf{1 . 8}$ & $\mathbf{9 8 . 5} \pm \mathbf{1 . 4}$ & $\mathbf{9 7 . 4} \pm \mathbf{1 . 8}$ & $\mathbf{9 7 . 4} \pm \mathbf{1 . 8}$ & $\mathbf{9 9 . 0} \pm \mathbf{1 . 4}$ & $75.4 \pm 8.4$ \\
CoSR+TSD & $\mathbf{9 8 . 0} \pm \mathbf{2 . 6}$ & $\mathbf{9 7 . 4} \pm \mathbf{1 . 8}$ & $\mathbf{9 8 . 5} \pm \mathbf{2 . 3}$ & $\mathbf{9 5 . 9} \pm \mathbf{2 . 3}$ & $\mathbf{9 7 . 4} \pm \mathbf{3 . 1}$ & $\mathbf{9 9 . 0} \pm \mathbf{1 . 4}$ & $\mathbf{8 4 . 6} \pm \mathbf{4 . 8}$ \\
\hline
\end{tabular}

* Average identification rates and standard deviations of different methods on Honda/UCSD dataset. Different sizes of the training and query image sets are considered in the experiments. For example, '50-15' means putting a restriction on the total number of images in the training and query sets with an upper limit of 50 and 15 frames respectively. The '-' in the last column means that the corresponding method can not be processed with only one image in each query set. The bold indicates the best for each column. sequence for each subject is randomly selected for training with the remaining three ones for testing. 10 runs of experiments are repeated for different random selections of the training and query sets. The average identification rates of all the experimented methods are exhibited in Table 2

From the table, we can find that the proposed method with both schemes achieves the highest average identification rate along with a relatively low standard deviation. This indicates that the proposed method is both accurate and stable. Among the other comparable methods, CHISD, SANP, MSSRC and RNP are based on affine hull or convex hull assumption while the earliest image set classification method MSM which earns a high identification rate represents different sets with a linear subspace. 
Table 2: Performance evaluation of all the methods on different datasets.

\begin{tabular}{cccc}
\hline Methods & Mobo & ETH & Scenes 15 \\
\hline MSM [1] & $96.8 \pm 2.0$ & $75.5 \pm 4.8$ & $\mathbf{1 0 0 . 0} \pm \mathbf{0 . 0}$ \\
DCC [3] & $92.8 \pm 1.2$ & $86.0 \pm 6.5$ & $96.7 \pm 2.4$ \\
MMD [4] & $92.5 \pm 2.9$ & $77.5 \pm 5.0$ & $98.7 \pm 1.8$ \\
MDA [5] & $81.0 \pm 12.3$ & $77.3 \pm 5.5$ & $98.0 \pm 3.0$ \\
AHISD [6] & $85.8 \pm 4.2$ & $78.8 \pm 5.3$ & $84.7 \pm 3.0$ \\
CHISD [6] & $96.4 \pm 0.8$ & $79.5 \pm 5.3$ & $86.7 \pm 3.3$ \\
SANP [8] & $\mathbf{9 7 . 5} \pm \mathbf{1 . 8}$ & $77.8 \pm 7.3$ & $99.3 \pm 1.5$ \\
CDL [14] & $90.0 \pm 5.0$ & $77.8 \pm 4.2$ & $86.7 \pm 0.0$ \\
MSSRC [13] & $95.6 \pm 1.8$ & $77.0 \pm 4.8$ & $90.7 \pm 2.8$ \\
SSDML [39] & $95.3 \pm 3.2$ & $81.9 \pm 6.6$ & $99.3 \pm 1.5$ \\
RNP [38] & $96.1 \pm 1.8$ & $81.0 \pm 3.2$ & $\mathbf{1 0 0 . 0} \pm \mathbf{0 . 0}$ \\
\hline CoSR+LB & $\mathbf{9 7 . 8} \pm \mathbf{2 . 1}$ & $\mathbf{9 1 . 5} \pm \mathbf{4 . 2}$ & $97.3 \pm 2.8$ \\
CoSR+TSD & $\mathbf{9 7 . 5} \pm \mathbf{1 . 5}$ & $\mathbf{9 2 . 0} \pm \mathbf{4 . 5}$ & $\mathbf{1 0 0 . 0} \pm \mathbf{0 . 0}$ \\
\hline
\end{tabular}

\subsubsection{ETH-80 Dataset}

For image set classification, ETH-80 dataset is a special one aiming at object categorization. It contains images of eight object categories including apples, cars, cows, cups, dogs, horses, pears and tomatoes. Each object category is formed by ten sub-categories such as different brands of cars or different breeds of dogs with images under 41 orientations. Following the experimental setup similar to that of [5], each subcategory is considered as an image set with 80 sets in total. From each object, five subcategories are randomly picked for training and the remaining five ones are taken for testing. We repeat the experiments for 10 times by taking different random selections of the training and query sets. As above, the average identification rates and standard deviations are presented in Table 2 ,

From the table, we will observe that the increase in performance of our proposed method against the other methods is more significant for this dataset. This is partly due to the fact that the objects in this dataset have more fine 
and structure information than the faces above. It is harder to model different objects well and makes the methods initially designed for face image set classification less effective. However, the good discriminant ability of sparse representation and complementary information from the cooperation of two opposite SRs make the proposed method suitable for distinguishing different objects with more fine characteristics.

\subsubsection{Scenes 15}

Different from traditional tasks of image set classification focusing on face

identification and object categorization, in this paper, we take scene classification into consideration and formulate it as an image set classification problem. We make use of Scenes 15 dataset to conduct our experiments. This dataset consists of totally 4485 images from 15 scene categories, with the number of images for each category ranging from 200 to 400. The 15 categories contain images from living room and kitchen to street and industrial. As this dataset is designed for single image based classification originally and contains complex scenes with many small objects, the intra and inter differences are more apparent than the tasks above. The experiments are conducted for 10 times as well and the average recognition rates are shown in Table 2

From the table, we can see that most of the methods can achieve a high recognition rate larger than $90 \%$. Considering the results of the state of the art single image based algorithms 28] for this dataset, we can reach the conclusion that scene classification task can also be modeled as an image set classification problem and better results can be obtained for the mutual information provided by the samples of the same set. Compared with the results on the other datasets, the better results on Scenes 15 may indicate that it is easier to explore potential common characteristics among inter sets and significant differences between intra sets with a discriminant feature (ScSPM). Also we can find that our proposed method can earn a promising result compared with the other methods. 
Table 3: Performance of 'CoSR+LB' scheme on various datasets with different additional dictionary size. $n$ represents the size of learned additional dictionary.

\begin{tabular}{cccccc}
\hline \multicolumn{2}{c}{$n$} & Honda & Mobo & ETH & Scenes 15 \\
\hline \multirow{2}{*}{ fSR } & $95.4 \pm 1.2$ & $95.8 \pm 2.6$ & $85.5 \pm 6.9$ & $94.7 \pm 1.8$ \\
\hline \multirow{2}{*}{1500} & bSR & $25.5 \pm 1.2$ & $83.3 \pm 2.2$ & $61.0 \pm 3.4$ & $70.0 \pm 3.3$ \\
& Fusion & $96.9 \pm 2.2$ & $\mathbf{9 7 . 8} \pm \mathbf{2 . 1}$ & $\mathbf{9 1 . 5} \pm \mathbf{4 . 2}$ & $94.7 \pm 1.8$ \\
\hline \multirow{2}{*}{1200} & bSR & $38.0 \pm 4.9$ & $82.5 \pm 3.3$ & $59.0 \pm 6.0$ & $72.0 \pm 3.0$ \\
& Fusion & $97.4 \pm 1.8$ & $96.9 \pm 2.3$ & $87.5 \pm 4.0$ & $96.0 \pm 2.8$ \\
\hline \multirow{2}{*}{900} & bSR & $74.4 \pm 6.3$ & $81.9 \pm 4.3$ & $61.0 \pm 1.4$ & $71.3 \pm 1.8$ \\
& Fusion & $\mathbf{9 8 . 5} \pm \mathbf{2 . 3}$ & $96.7 \pm 2.3$ & $89.5 \pm 2.7$ & $96.0 \pm 3.7$ \\
\hline \multirow{2}{*}{600} & bSR & $86.7 \pm 4.6$ & $82.5 \pm 3.5$ & $58.0 \pm 3.7$ & $66.0 \pm 4.9$ \\
& Fusion & $96.9 \pm 2.2$ & $97.5 \pm 0.6$ & $87.5 \pm 5.6$ & $\mathbf{9 7 . 3} \pm \mathbf{2 . 8}$ \\
\hline
\end{tabular}

\subsection{Ablative Analysis}

Here we present an ablative analysis in order to analyze different components of our model with two distinct schemes to learn dictionaries for bSR.

\subsubsection{Effects of Parameters}

There is a parameter to control the size of learned dictionary, which will affect the discriminant ability of the learned dictionary. For 'Co+LB', it is the size $n$ of additional bases in Eqn. (16) and for 'Co+TSD', it is the ratio $\gamma$ between $N_{\mathcal{D}_{1}}$ and $N_{\mathcal{D}}$ in Alg.(1). The results on all the evaluated datasets for different settings of these two parameters are shown in Table 3 and 4 respectively.

From the tables, we can notice the following remarks:

1) for both schemes, combining respective decisions from two opposite directions, namely fSR and bSR, the performance will be improved as expected. It indicates that there is complementary information between these two SRs and their procedures are conditionally independent.

6002 ) for different $\gamma$ and $n$, the performance of bSR is different definitely but keeps 
Table 4: Performance of 'CoSR+TSD' scheme on various datasets with different $\gamma \cdot \gamma$ stands for the ratio between $N_{\mathcal{D}_{1}}$ and $N_{\mathcal{D}}$.

\begin{tabular}{|c|c|c|c|c|c|}
\hline & $\gamma$ & Honda & Mobo & ETH & Scenes 15 \\
\hline & fSR & $94.9 \pm 0.0$ & $95.8 \pm 2.6$ & $88.5 \pm 3.8$ & $95.3 \pm 1.8$ \\
\hline \multirow{3}{*}{0.5} & bSR & $94.4 \pm 2.8$ & $76.9 \pm 2.5$ & $86.5 \pm 3.8$ & $99.3 \pm 1.5$ \\
\hline & Fusion & $97.4 \pm 2.6$ & $96.7 \pm 1.6$ & $89.0 \pm 4.2$ & $98.7 \pm 1.8$ \\
\hline & SCI & $\mathbf{9 8 . 0} \pm \mathbf{2 . 8}$ & $96.7 \pm 2.1$ & $90.5 \pm 3.3$ & $99.3 \pm 1.5$ \\
\hline \multirow{3}{*}{0.7} & bSR & $95.4 \pm 3.3$ & $95.0 \pm 0.8$ & $87.0 \pm 4.8$ & $99.3 \pm 1.5$ \\
\hline & Fusion & $96.9 \pm 2.8$ & $96.7 \pm 2.1$ & $90.0 \pm 4.0$ & $98.7 \pm 1.8$ \\
\hline & $\mathrm{SCI}$ & $97.4 \pm 2.6$ & $97.2 \pm 2.2$ & $92.0 \pm 4.5$ & $100.0 \pm 0.0$ \\
\hline \multirow{3}{*}{0.8} & bSR & $93.9 \pm 3.9$ & $96.9 \pm 1.8$ & $89.0 \pm 3.4$ & $99.3 \pm 1.5$ \\
\hline & Fusion & $96.9 \pm 2.2$ & $97.5 \pm 1.5$ & $91.0 \pm 2.2$ & $98.7 \pm 1.8$ \\
\hline & $\mathrm{SCI}$ & $97.4 \pm 2.6$ & $97.5 \pm 1.5$ & $91.0 \pm 2.0$ & $99.3 \pm 1.5$ \\
\hline \multirow{3}{*}{0.9} & bSR & $91.3 \pm 2.9$ & $96.7 \pm 0.8$ & $88.5 \pm 1.4$ & $97.3 \pm 2.8$ \\
\hline & Fusion & $95.9 \pm 1.4$ & $96.9 \pm 1.8$ & $91.0 \pm 4.9$ & $98.0 \pm 3.0$ \\
\hline & SCI & $96.9 \pm 2.2$ & $96.9 \pm 1.8$ & $91.0 \pm 2.9$ & $99.3 \pm 1.5$ \\
\hline
\end{tabular}

relatively stable except for the abnormal case in Honda dataset for 'CoSR+LB' scheme. This may be caused by too few images in some concepts and a large size of dictionary will lead to a low discriminant ability. And the changes of fusion results also stay in an acceptable scope, which demonstrates the stability of our proposed method.

3) for 'CoSR+TSD' scheme, the additional SCI reweighing procedure is helpful for correcting wrong biased independent classifiers and therefore can improve the fusion performance. And it is more apparent for ETH-80 and Scenes 15 dataset. The relatively low improvements in Honda and Mobo dataset may be due to the good discriminant nature of sparse representation for face recognition. This will push the coefficients to concentrate on only a few concepts and 
makes the SCI term invalid.

4) overall, the performance of 'CoSR+TSD' scheme is better than that of 'CoS$\mathrm{R}+\mathrm{LB}$ ' scheme. This may be due to the fact that the dictionary produced by 'CoSR+TSD' scheme originates from the existing natural images and contains more discriminant information than the artificial one produced by 'CoSR+LB' scheme.

\subsubsection{Dictionary Validation}

In this subsection, we will provide a validation for the quality of learned dictionary produced by 'Learning Bases'. For both 'learned bases' and 'random bases', their recognition rates are exhibited in Table 5 and time consumption for testing is shown in Table 6. The size of dictionary is set to 600 for both schemes.

Table 5: The comparison of recognition rates (\%) between random bases and learned bases. Bold indicates the best for each column.

\begin{tabular}{cccccc}
\hline \multicolumn{2}{c}{ Schemes } & Honda & Mobo & ETH & Scenes 15 \\
\hline \multirow{2}{*}{ Random } & bSR & $80.5 \pm 4.9$ & $70.2 \pm 3.0$ & $48.5 \pm 6.8$ & $10.7 \pm 2.8$ \\
& Fusion & $95.4 \pm 1.8$ & $95.5 \pm 3.3$ & $86.0 \pm 2.8$ & $95.0 \pm 3.0$ \\
\hline \multirow{2}{*}{ Learned } & bSR & $91.8 \pm 1.2$ & $82.2 \pm 3.3$ & $59.0 \pm 2.4$ & $66.0 \pm 4.9$ \\
& Fusion & $\mathbf{9 6 . 9} \pm \mathbf{1 . 2}$ & $\mathbf{9 7 . 5} \pm \mathbf{0 . 6}$ & $\mathbf{8 8 . 0} \pm \mathbf{4 . 0}$ & $\mathbf{9 7 . 3} \pm \mathbf{1 . 8}$ \\
\hline
\end{tabular}

Table 6: The comparison of time consumption for testing (in seconds) between random bases and learned bases.

\begin{tabular}{lcccc}
\hline Schemes & Honda & Mobo & ETH & Scenes 15 \\
\hline Random & 11.3 & 13.2 & 8.4 & 5.5 \\
\hline Learned & 5.8 & 6.5 & 4.3 & 3.1 \\
\hline
\end{tabular}

From Table 5, we can find that with the help of 'learned bases', better results are obtained for bSR and accordingly the fusion perfor- 
mance is improved. Meanwhile, after the period of dictionary training, the time used to recognize a query set is reduced (shown in Table 6), which is quite important for image set classification task. All these results demonstrate the effectiveness and necessity of learned bases.

${ }_{630}$ 5.3.3. Comparison Between Different Schemes for Training Sets Division

In this subsection, we will provide a comparison for different schemes to form the additional bases for 'Training Sets Division'. For both schemes, their recognition rates are exhibited in Table $7, \gamma$ is set to 0.5 and the schemes in Sec.(4.3) are both applied.

Compared with the results in Table 4, we can find that for both schemes, lower recognition rates are obtained during the period of bSR if the schemes in Sec.(4.3) are applied. This is due to the fact that the number of training samples is greatly reduced. However, much better results are obtained for 'HDC'. When it turns to the period of fusion, the CoSR model reduces to traditional fSR model for 'Random Selection' as little information is gained from degraded bSR. However, the results of 'HDC' are affected only a little, which indicates that most of the complementary information for fSR is retained. The abnormal case for Scenes 15 Dataset may be due to the good discriminant ability of the features (ScSPM). All these results demonstrate the effectiveness of the adopted schemes.

\subsubsection{Timing Analysis}

A comparison of computational complexity of all the methods on the benchmark ETH-80 dataset using a Core 4 Quad Machine is presented in Table 8. The schemes in Sec.44.3) are both applied to improve the efficiency (the speedup rates for 'Compression' and ' $\mathrm{KNN}$ ' are 5 and 3 respectively here). $\gamma$ is set to 0.5 and the dictionary size $n$ takes the value of 600 .

From the table, the following remarks can be noticed. For 'Co+LB', 
Table 7: The comparison of recognition rates (\%) between 'Random Selection' and 'HDC' for 'Co+TSD'. 'Random Selection' means randomly picking out a certain number of samples to form $\mathcal{D}_{1}$ and $\mathcal{D}_{2}$. Bold indicates the best for each column.

\begin{tabular}{cccccc}
\hline \multicolumn{2}{c}{ Schemes } & Honda & Mobo & ETH & Scenes 15 \\
\hline \multirow{2}{*}{ Random } & bSR & $8.2 \pm 2.2$ & $30.6 \pm 4.6$ & $12.5 \pm 0.0$ & $62.0 \pm 6.9$ \\
& Fusion & $94.9 \pm 0.0$ & $95.8 \pm 2.6$ & $89.5 \pm 2.1$ & $97.3 \pm 3.3$ \\
\hline \multirow{2}{*}{ HDC } & bSR & $93.9 \pm 1.2$ & $75.8 \pm 0.8$ & $85.5 \pm 5.3$ & $76.7 \pm 6.7$ \\
& Fusion & $\mathbf{9 7 . 4} \pm \mathbf{1 . 8}$ & $\mathbf{9 7 . 2} \pm \mathbf{2 . 1}$ & $\mathbf{9 1 . 5} \pm \mathbf{3 . 4}$ & $\mathbf{9 8 . 7} \pm \mathbf{1 . 8}$ \\
\hline
\end{tabular}

there is an additional training stage to learn the discriminant bases, which can be performed offline. For 'Co+TSD', there is not any time spent on training as the samples in the training data are taken directly to form the additional bases. When it turns to the testing period, 'Co+LB' owns a smaller time consumption than 'Co+TSD'. Although our model needs to spend more time to identify each set than the methods such as MSM, MDA, RNP, its time is less or comparable to those based on sparse representation (MSSRC and SANP). And the time is acceptable with high recognition rates and a low expense on training.

Table 8: A comparison of time consumption (in seconds) for training the models and identifying a query set for all the methods.

Methods MSM DCC MMD MDA AHISD CHISD SANP CDL RNP MSSRC SSDML Co+LB Co+TSD

\begin{tabular}{c|ccccccccccccc}
\hline Train & $\mathrm{N} / \mathrm{A}$ & 13.36 & $\mathrm{~N} / \mathrm{A}$ & 1.22 & $\mathrm{~N} / \mathrm{A}$ & $\mathrm{N} / \mathrm{A}$ & $\mathrm{N} / \mathrm{A}$ & 76.21 & $\mathrm{~N} / \mathrm{A}$ & $\mathrm{N} / \mathrm{A}$ & 21.92 & 20.18 & $\mathrm{~N} / \mathrm{A}$ \\
Test & .045 & .311 & 8.43 & .005 & .095 & .213 & 105.7 & 1.40 & .027 & 4.78 & .577 & 3.12 & 4.26 \\
\hline
\end{tabular}


In this paper, we focus on image set classification where the query samples are modeled into a set and provide plenty of highlights for fields including face recognition, object categorization, etc. As there may exist the situation that the set size is small, a discriminant model which can find out more hidden information from limited set samples should be proposed. Considering the problem ahead and the complementary information provided from the cooperation between fSR and bSR, we extend the cooperative sparse representation (CoSR) model to the field of image set classification. It is achieved by proposing two different schemes, namely 'Learning Bases' and 'Training Sets Division', to construct additional discriminant dictionary to cooperate with samples in query set and fulfill the procedure of bSR. And with the fusion of the decisions from these two SRs, the performance of the CoSR model is improved. The promising results obtained on several benchmark datasets also demonstrate the effectiveness of our method. At the same time, we model scene classification task as 680 a member of image set classification, which has not been declared so far, and show that better results can be obtained via existing methods in this field.

There are still many open issues in the future. For example, a method to divide training sets into two subsets with balanced and representative samples is meaningful, and the way to learn more discriminant and common bases shared by all the concepts is of significance.

\section{Acknowledgments}

This research was supported by the National Natural Science Foundation of China (No. 61375047), the 973 Program of China (No.2013CB329604), the Program for Changjiang Scholars and Innovative Research Team in University of the Ministry of Education of China (No.IRT13059), the US National Science Foundation (NSF CCF-0905337), the Hong Kong Scholars Program (No.XJ2012012), and the Fundamental Research Funds for the Central Universities of China. 


\section{References}

[1] W. Zhao, R. Chellappa, P. J. Phillips, A. Rosenfeld, Face recognition: A literature survey, ACM computing surveys (CSUR) 35 (4) (2003) 399-458.

[2] A. S. Mian, M. Bennamoun, R. Owens, An efficient multimodal 2d-3d hybrid approach to automatic face recognition, IEEE Transactions on Pattern Analysis and Machine Intelligence 29 (11) (2007) 1927-1943.

[3] T.-K. Kim, J. Kittler, R. Cipolla, Discriminative learning and recognition of image set classes using canonical correlations, IEEE Transactions on Pattern Analysis and Machine Intelligence 29 (6) (2007) 1005-1018.

[4] R. Wang, S. Shan, X. Chen, W. Gao, Manifold-manifold distance with application to face recognition based on image set, in: Computer Vision and Pattern Recognition, IEEE, 2008, pp. 1-8.

[5] R. Wang, X. Chen, Manifold discriminant analysis, in: Computer Vision and Pattern Recognition, IEEE, 2009, pp. 429-436.

[6] H. Cevikalp, B. Triggs, Face recognition based on image sets, in: Computer Vision and Pattern Recognition (CVPR), IEEE, 2010, pp. 2567-2573.

[7] M. T. Harandi, C. Sanderson, S. Shirazi, B. C. Lovell, Graph embedding discriminant analysis on grassmannian manifolds for improved image set matching, in: Computer Vision and Pattern Recognition (CVPR), IEEE, 2011, pp. 2705-2712.

[8] Y. Hu, A. S. Mian, R. Owens, Face recognition using sparse approximated nearest points between image sets, IEEE Transactions on Pattern Analysis and Machine Intelligence 34 (10) (2012) 1992-2004.

[9] M. Hayat, M. Bennamoun, S. An, Learning non-linear reconstruction models for image set classification, in: Computer Vision and Pattern Recognition (CVPR), IEEE, 2014, pp. 1915-1922. 
[10] M. Hayat, M. Bennamoun, S. An, Reverse training: An efficient approach for image set classification, in: ECCV, Springer, 2014, pp. 784-799.

[11] O. Yamaguchi, K. Fukui, K.-i. Maeda, Face recognition using temporal image sequence, in: Third IEEE International Conference on Automatic Face and Gesture Recognition, IEEE, 1998, pp. 318-323.

[12] J. Lu, G. Wang, P. Moulin, Image set classification using holistic multiple order statistics features and localized multi-kernel metric learning, in: IEEE International Conference on Computer Vision (ICCV), IEEE, 2013, pp. $329-336$.

[13] E. G. Ortiz, A. Wright, M. Shah, Face recognition in movie trailers via mean sequence sparse representation-based classification, in: Computer Vision and Pattern Recognition (CVPR), IEEE, 2013, pp. 3531-3538.

[14] R. Wang, H. Guo, L. S. Davis, Q. Dai, Covariance discriminative learning: A natural and efficient approach to image set classification, in: Computer Vision and Pattern Recognition (CVPR), IEEE, 2012, pp. 2496-2503.

[15] B. A. Olshausen, D. J. Field, Sparse coding with an overcomplete basis set: A strategy employed by v1?, Vision research 37 (23) (1997) 3311-3325.

[16] J. Wright, A. Y. Yang, A. Ganesh, S. S. Sastry, Y. Ma, Robust face recognition via sparse representation, IEEE Transactions on Pattern Analysis and Machine Intelligence 31 (2) (2009) 210-227.

[17] J. Yang, K. Yu, Y. Gong, T. Huang, Linear spatial pyramid matching using sparse coding for image classification, in: CVPR, IEEE, 2009, pp. $1794-1801$.

[18] Y. Liu, F. Wu, Y. Zhuang, Group sparse representation for image categorization and semantic video retrieval, Science China Information Sciences 54 (10) (2011) 2051-2063. 
[25] M. T. Harandi, C. Sanderson, A. Wiliem, B. C. Lovell, Kernel analysis over riemannian manifolds for visual recognition of actions, pedestrians and textures, in: IEEE Workshop on Applications of Computer Vision (WACV), IEEE, 2012, pp. 433-439.

[26] S. Lazebnik, C. Schmid, J. Ponce, Beyond bags of features: Spatial pyramid 770

[19] H. Liu, F. Sun, M. Gao, Visual tracking using iterative sparse approximation, in: Advances in Neural Networks-ISNN 2011, Springer, 2011, pp. $207-214$.

[20] S. Chen, C. Sanderson, M. T. Harandi, B. C. Lovell, Improved image set classification via joint sparse approximated nearest subspaces, in: Computer Vision and Pattern Recognition (CVPR), IEEE, 2013, pp. 452-459.

[21] Z.-Q. Zhao, H. Glotin, Z. Xie, J. Gao, X. D. Wu, Cooperative sparse representation in two opposite directions for semi-supervised image annotation, IEEE Transactions on Image Processing 21 (9) (2012) 4218-4231.

[22] O. Arandjelović, G. Shakhnarovich, J. Fisher, R. Cipolla, T. Darrell, Face recognition with image sets using manifold density divergence, in: Computer Vision and Pattern Recognition (CVPR), Vol. 1, IEEE, 2005, pp. $581-588$.

[23] P. Turaga, A. Veeraraghavan, A. Srivastava, R. Chellappa, Statistical computations on grassmann and stiefel manifolds for image and video-based recognition, IEEE Transactions on Pattern Analysis and Machine Intelligence 33 (11) (2011) 2273-2286.

[24] J. Hamm, D. D. Lee, Grassmann discriminant analysis: a unifying view on subspace-based learning, in: Proceedings of the 25th international Conference on Machine learning, ACM, 2008, pp. 376-383.

matching for recognizing natural scene categories, in: CVPR, 2006, pp. 2169-2178. 
[27] L.-J. Li, H. Su, L. Fei-Fei, E. P. Xing, Object bank: A high-level image representation for scene classification \& semantic feature sparsification, in: Advances in neural information processing systems, 2010, pp. 1378-1386.

[36] P. Viola, M. J. Jones, Robust real-time face detection, International journal of omputer vision 57 (2) (2004) 137-154. 
[37] D. Ciresan, U. Meier, L. M. Gambardella, J. Schmidhuber, Deep big simple neural nets excel on handwritten digit recognition, Corr 22 (12) (2010) $3207-3220$.

[38] M. Yang, P. Zhu, L. Van Gool, L. Zhang, Face recognition based on regularized nearest points between image sets, in: 2013 10th IEEE International Conference and Workshops on Automatic Face and Gesture Recognition (FG), IEEE, 2013, pp. 1-7.

[39] P. Zhu, L. Zhang, W. Zuo, D. Zhang, From point to set: Extend the learning of distance metrics, in: 2013 IEEE International Conference on Computer Vision (ICCV), IEEE, 2013, pp. 2664-2671.

Peng Zheng is a Ph.D candidate at Hefei University of Technology since 2010. He received his Bachelor's degree in 2010 from Hefei University of Technology. His interests cover pattern recognition, image processing and computer vision.

Zhong-Qiu Zhao is a professor at Hefei University of Technology, China. He obtained the Master's degree in Pattern Recognition \& Intelligent System at Institute of Intelligent Machines, Chinese Academy of Sciences, Hefei, China, in 2004, and the PhD degree in Pattern Recognition \& Intelligent System at University of Science and Technology, China, in 2007. From April 2008 to November 2009, he held a postdoctoral position in image processing in CNRS UMR6168 Lab Sciences de l'Information et des Systèmes, France. From January 2013 to December 2014, he held a research fellow position in image processing at the Department of Computer Science of Hongkong Baptist University, Hongkong, China. Now he works in Laboratory of Data Mining and Intelligent Computing, Hefei University of Technology, China. His research is about pattern recognition, image processing, and computer vision. 
Jun Gao was born in 1963 and obtained his Bachelor's and Master's degree from Hefei University of Technology in 1985 and 1990. After that, he received his Ph.D. degree at Chinese Academy of Sciences in 1999. Now he is a professor and Ph.D. supervisor in the School of Computer and Information at Hefei University of Technology. His research interests include image processing, computer vision and intelligent information processing.

Xindong $\mathbf{W u}$ is a professor of Computer Science at the University of Vermont ${ }_{830}$ (USA), a Yangtze River Scholar in the School of Computer Science and Information Engineering at the Hefei University of Technology (China), and a Fellow of the IEEE and the AAAS.

Dr. Wu received his Bachelors and Masters degrees in Computer Science from Hefei University of Technology, China, and his Ph.D. degree in Artificial 835 Intelligence from the University of Edinburgh, Britain. His research interests include data mining, knowledge-based systems, and Web information exploration. $\mathrm{He}$ is the Steering Committee Chair of the IEEE International Conference on Data Mining (ICDM) the Editor-in-Chief of Knowledge and Information Systems (KAIS, by Springer), and a Series Editor of the Springer Book Series on 840 Advanced Information and Knowledge Processing (AI\&KP). He was the Editorin-Chief of the IEEE Transactions on Knowledge and Data Engineering (TKDE, by the IEEE Computer Society) between 2005 and 2008. He served as Program Committee Chair/Co-Chair for ICDM 03 (the 2003 IEEE International Conference on Data Mining), KDD-07 (the 13th ACM SIGKDD Interna-tional 845 Conference on Knowledge Discovery and Data Mining), and CIKM 2010 (the 19th ACM Conference on Information and Knowledge Management). 